Vorgesetzten gemeldet wird (unabhängig vom Meldesystem) kann das Disziplinarsystem aktiv werden.

Die Hintergrundinformationen zu gemeldeten Vorfällen und die von den beteiligten Flugzeugführern erläuterten beitragenden Faktoren verdeutlichen immer wieder, dass häufig private $\mathrm{Be}$ lastungen der Auslöser für gravierende, fliegerische Arbeitsfehler sind. Ein stabiles privates Umfeld ist eine wichtige Voraussetzung für hohe berufliche Leistungsfähigkeit. Ein Pilot wird - sofern er seine privaten Probleme offenbart - unter Beibehaltung seiner Bezüge, vorübergehend vom fliegerischen Dienst freigestellt, bis sich seine Lebenssituation wieder beruhigt hat.

Die Erwartung, dass die Hochtechnologie Fachkenntnis und gesunden Menschenverstand weitgehend überflüssig macht, hat sich nicht erfüllt. Es ist beinahe ein Treppenwitz der Menschheitsgeschichte, dass die Bemühungen des Menschen, Maschinen zu entwickeln, die menschliche Schwächen kompensieren, dazu geführt haben, dass nun beim Umgang mit diesen HITEC-Geräten so- ziale Kompetenz und optimale Teamarbeit, also „urmenschliche“ Fähigkeiten einen ganz besonderen Stellenwert gewonnen haben.

\author{
Manfred Müller \\ Flugkapitän \\ Leiter Flugsicherheit Lufthansa \\ Dozent für Risikomanagement \\ Hochschule Bremen \\ E-Mail: manfred.mueller@akarisma.de
}

\title{
Der Arzt als Sicherheitsrisiko? - Ein Kommentar
}

\author{
Es lassen sich einige Parallelen zwischen dem Berufsbild eines Piloten und dem eines Mediziners erkennen. Doch \\ lassen sich Sicherheitsstrategien wie die aus der Luftfahrt tatsächlich in die medizinische Praxis übertragen? Dies \\ wäre ein weiter Weg, an dessen Anfang ein Umdenken stünde.
}

Ü berall, wo Menschen mit Technik arbeiten, also auch im Gesundheitswesen kann es zu Fehlern kommen, die in Katastrophen münden. Im Falle der Medizin ist beispielsweise das Narkosegerät nur so gut, wie es durch den Anästhesisten bedient wird, vergleichbar mit einem Flugzeug, das ein Pilot fliegt. Durch Kontrolle und Kooperation von Ärzten und Pflegern können Fehler reduziert und Katastrophen vermieden werden.

Zum Beispiel können elektive Eingriffe, insbesondere operativer Art, die in der Vorbereitung hohe Standards in der Medizin erforderlich machen, das Risiko des Eingriffes insgesamt minimieren und dementsprechend das Gesundheitsrisiko für den Patienten senken.

Im Beitrag von Manfred Müller wurde bereits kurz auf die Gemeinsamkeiten zwischen einem Arzt und einem Flugkapitän hinsichtlich der Verantwortung, die sie tragen, hingewiesen. Für die Medizin müsste die Konsequenz daraus lauten, dass sich von vornherein immer zwei Anästhesisten oder ein Anästhesist und ein Anästhesiepfleger permanent um den Patienten kümmern. Dies entspricht leider nicht der Realität im Operationssaal. Häufig ist der Anästhesist oder der Pfleger alleine im Operationssaal und versorgt den Patienten.
Erst wenn es zu Problemen (Komplikation OPS) kommt, wird ein weiterer Anästhesist oder ein Pfleger hinzugezogen. Dies führt zwangsläufig zu einem Zeitfenster, in dem der Patient einem erhöhten Risiko ausgesetzt ist.

Für eine verbesserte Patientensicherheit müssten Arbeitsstrukturen geschaffen werden, um Fehler und Probleme früher zu erkennen und Lösungen eher angehen zu können. Um dies zu gewährleisten, wäre folgerichtig eine bessere Personalausstattung zu fordern. Führen wir uns unser Gesundheitssystem mit seinen aktuellen Bedingungen vor $\mathrm{Au}$ gen, wird klar, dass dies nicht realistisch durchführbar ist.

Warum? Erstens wäre das erhöhte Personalaufkommen - beispielsweise im Operationssaal - für die Krankenhäuser im aktuellen Vergütungssystem nicht finanzierbar. Zweitens fehlt es unter den heutigen Bedingungen schlicht an gut ausgebildetem Fachpersonal etwa zur Durchführung von Narkosen. Nicht ohne Grund haben viele Krankenhäuser vermehrt „Leihärzte“ in ihren Reihen, da der Markt nicht mehr Fachärzte hergibt.

Flugkapitän Müller hat vollkommen Recht, wenn er eine flache Hierarchie mit flachen Gradienten fordert. In der heutigen Struktur des Krankenhauswe- sens ist dies allerdings mehr Wunsch als Wirklichkeit. Sicherlich gibt es Abteilungen in der diese flache Hierarchie gelebt wird, ich selbst habe im Operationssaal aber noch nie erlebt, dass ein Assistenzarzt dem Chef- oder Oberarzt das Skalpell aus der Hand nahm und die Operation weiterführte. Hier wäre das Umdenken aller Beteiligten, sowohl der Chef- und Oberärzte als auch der OPSchwestern und Pfleger sicherlich ein erster Schritt. Ließe sich im Operationssaal eine Atmosphäre kreieren, in der niemand aufgrund seiner Stellung Hemmungen hat, ebenfalls auf die Sicherheit zu achten und gegebenenfalls Zweifel zu äußern, könnten wir uns den aktuellen Sicherheitsstandards der Luftfahrt annähern. Aktuell ist uns die Luftfahrt hinsichtlich der Risikominimierung aber noch um Längen voraus.

\footnotetext{
Norbert Schürmann

Facharzt Anästhesie, Allgemeinmedizin

Departmentleiter der Abteilung für

Schmerz-und Palliativmedizin

Abteilung für Anästhesie,

St. Josef Krankenhaus Moers

E-Mail: pal.schuermann@st-josef-moers.de
} 